\title{
Feeling like a Fraud: Imposter Syndrome in Political Science
}

\section{Imposter Syndrome: What are the signs?}

Many prospective and current political science graduate students experience imposter syndrome; however, imposter syndrome is not experienced equally or in a universal manner. Intersectional aspects of identity, including gender, nationality, and race, can also shape and compound how imposter syndrome is experienced. Given this, we distinguish between two modes of imposter syndrome: (i) feelings of fooling others; and (ii) anxiety from having to perform. But, broadly speaking, what is imposter syndrome? How do we characterize it? There are many assumptions about imposter syndrome, such as someone who does not want to work hard, or has feelings of not belonging, among other feelings of self-worth and anxiety. However, imposter syndrome is a psychological term that denotes a "pattern of behavior wherein people (even those with adequate external evidence of success) doubt their abilities and have a persistent fear of being exposed as a fraud" (Mullangi and Jagsi 2019, 403). Others have similarly defined this concept, noting that it is a very "real and specific form of intellectual self-doubt" that can feature anxiety and depression (Weir 2013), fear of judgment by colleagues (Evans et al. 2018, 283), and underestimation of "talents by ignoring evidence for those talents" (Slank 2019, 206).

The first mode of imposter syndrome - feelings of fooling others - can be characterized mostly by feeling like a fraud, feeling a lack of belonging to the department, believing that "success was obtained through luck," and fearing being "found out" to be an imposter (Slank 2019, 205-209). Within this, there is typically a concern that people perceived to be important will discover that graduate students (that feel like imposters) are not "capable" (Slank 2019, 209). Some of the feelings of fooling others comes from a lack of evidence about what others know about you but that you have full knowledge of, meaning you have evidence of your imperfection but others do not. A colloquialism to this feeling is "fake it "til you make it," meaning to act as though you understand what you are doing and saying in a setting until you feel like you belong in that setting. Anxiety can stem from the belief that others will see through your fake persona and see that you are actually not capable of graduate work or the ability to work in the discipline.

The second mode of imposter syndrome - anxiety from having to perform - is more closely associated with the realization that graduate students are expected to immerse themselves in the norms of the political science discipline, and the engagement that comes with this. For example, a graduate student may be invited to co-author with a member of faculty or be accepted to present at a conference. In these instances, the graduate student may experience self-doubt and be overwhelmed by these newfound responsibilities that can appear daunting. Fear of not meeting the expectations of a mentor, professor, or of members of the profession can hinder the ability to perform activities associated with being a member of the profession, such as writing, presenting, researching, and producing information to different audiences such as peers, students, or the public. This mode of imposter syndrome, while closely tied to the first, can be debilitating and lead to negative health and professional outcomes. For this reason, we choose to distinguish between these two modes - acknowledging that in some sense we all "feel like imposters," but some people experience this feeling more acutely and with more dire consequences.

Within both of these modes, graduate students in political science are prone to compare themselves to others, and these comparisons can "foster self-doubt" and lead to "skewed" perceptions of 
oneself and others (Bothello and Roulet 2019, 857). Broadly, graduate students are also more than "six times as likely to experience depression and anxiety" compared to the general population, thereby exacerbating feelings associated with imposter syndrome (Evans et al. 2018, 282). In turn, these instances of self-doubt, skewed perceptions, and poor mental health can have significant impacts on graduate students' capacity to learn, conduct research, teach (as an instructor or teaching assistant), engage with colleagues, and pursue professional development. To combat the effects of imposter syndrome then, graduate students must more openly discuss mental health challenges associated with these areas of development (Hummel and Kurd 2021), as well as re-examine their workload so as to prevent burnout and further self-doubt and comparisons between oneself and others (Muller 2020, 1280).

\section{Impact of Imposter Syndrome on Graduate Students}

As indicated in the preceding section, imposter syndrome can adversely affect mental and physical health as well as the capacity to operate effectively in the discipline of political science - as a student, research or teaching assistant, instructor, and colleague. However, not everyone experiences imposter syndrome and even among those who do, the experiences are not necessarily identical. Further, imposter syndrome is not limited to prospective and current graduate students, but can also be found among post-doctoral students, and even tenured faculty. This also means that imposter syndrome can 'look' differently at different stages of an academic career in political science. For example, prospective students may first consider how their academic backgrounds differ from their peers and that they feel less 'worthy' in the program. For post-candidacy students, it may be about how their peers have managed to publish a journal article while they have not. For those students on the job market, it may be a perception that peers are receiving more interest for their work along with a deep, but reasonable, fear that a longterm career in this field is just not viable. Graduate students who are women, gender minorities, racial minorities, international students, or from other populations which are underrepresented in political science are also more likely to experience imposter syndrome due to the additional challenges (e.g., discrimination, harassment, visas) they face given these intersecting aspects of their identity (Slack 2019, 207; Mullangi and Jagsi 2019, 403). It is also harder for these students to disambiguate between real or perceived negative feedback which arises primarily from discrimination, verses that which is actually warranted. Such disciplinary gaslighting can fuel fears of imposter syndrome as students worry that they are overthinking the role of discriminatory feedback and worry that their work may indeed deserve such negative reception.

Given the disparities in gendered and cultural norms, these particular underrepresented groups of graduate students may also suffer from imposter syndrome in silence out of concern for stigmatization, discrimination, and "potential damage to their careers" (Muller 2020, 1280). This may also result in a lack of pursuit of departmental opportunities for professional development, academic service commitments, or collaboration "due to feeling unqualified" (Mullangi and Jagsi 2019, 403). Consequently, those experiencing imposter syndrome can fall into a spiral of self-doubt, constantly comparing themselves to others, and questioning the "value" of their profession and status (Bothello and Roulet 2019, 858). Such a spiral can result in poor work performance, "lack of response to emails, unexplained non-attendance, [and] sudden indecisiveness and unreliability" (Muller 2020, 1280) and possibly exit from the profession. In these instances, imposter syndrome can appear unescapable, sometimes leading students to spiral out of their graduate program and left feeling deflated at their perceived inadequacy. 
Imposter syndrome is particularly common among high-achieving people. In dealing with imposter syndrome, people can experience anxiety, dysphoric moods, emotional instability, negative selfevaluations, and perfectionism (Rohrmann, Bechtoldt, and Leonhardt 2016; Wilson and Cutri 2019, 72). In addition to these effects, students may engage in behavior that, to some extent, can sabotage their success. For example, students may feel inclined to set high expectations for themselves and feel disappointed when they fail to meet these expectations. Even when success is recognized by others faculty, peers, or family - there is a tendency for those experiencing imposter syndrome to downplay this evidence as insignificant. In settings outside academia, family, peers, and friends can introduce a mismatch of expectations, which can exacerbate feelings of being a fraud, lack of belonging, and anxiety to meet expectations from family, and friends outside academia.

Thus, the lack of self-confidence associated with imposter syndrome and the vicious cycle of selfdoubt can result in students refraining from grasping opportunities that allow for academic and professional development. These may include not collaborating with faculty, avoiding on- or off-campus volunteering, not applying for fellowships or grants, and not applying to or attending conferences to present academic research. Moreover, those experiencing imposter syndrome may withhold information during meetings (not talking in graduate courses), engage in delay tactics when asked to perform (e.g., procrastinating when it comes to grading, completing assignments, meeting faculty), and make selfdeprecatory comments. Breaking this cycle is undoubtedly challenging and fretting about breaking this cycle can only often lead to heightened psychological stress, whereby students engage in a culture of overwork and in behavior that results in burnout. Experiences of burnout, increased anxiety, loss of interest in hobbies, and poor sleep are likely symptoms that it is time for change. Given this, the next section focuses on how political science graduate students can best prevent or navigate existing experiences of imposter syndrome.

\section{What to do about Imposter Syndrome?}

To manage or help others manage imposter syndrome, a number of recommendations are provided here. It is crucial that political science graduate students recognize what imposter syndrome is and remember that it can be experienced differently between people. Additionally, imposter syndrome should be normalized, not trivialized - this is a call for greater understanding that many people across the discipline of political science experience imposter syndrome but, at the same time, it should not be belittled as "everyone experiences it, [so it does not matter]" or downplayed as "it is just a process everyone goes through." As previously noted, there can be mental health concerns affiliated with imposter syndrome and it can seriously affect productivity and feelings of self-worth. Given this, imposter syndrome needs to be taken seriously.

It is also key to recognize the role that political science graduate students play in affecting imposter syndrome, should they themselves be experiencing it or seeking to prevent it. This begins with an understanding that departmental culture can strongly impact imposter syndrome. For example, an environment that encourages thinking about talent or capability or intelligence as malleable and teachable (Slank 2019, 214) may encourage better working conditions that halt the onset or development of imposter syndrome. As part of this, establishing a healthy work-life balance is crucial (Evans et al. 2018, 283), as is positive self-talk and affirmation (to combat feelings of self-doubt), and recognition of talents and achievements to realize what 'success' looks like and that success is not necessarily a fixed goal or a 
binary. In many political science graduate programs, students are often informed that they will learn to perform as a political scientist, and this can lead students to develop a binary understanding of success. This happens when students perceive the outcome of being considered a political scientist a great contrast to their current standing - whether a prospective, pre-candidacy, or post-candidacy student - when, instead, they should understand that becoming a political scientist is a journey that begins with being admitted to a political science graduate program. It is a gradual process in which students will acquire a range of skills and talents, develop their knowledge, and adhere to the norms of the discipline.

Political science graduate students should also seek to collaborate with and uplift their peers. Although competition can be healthy, discussing class material with peers, co-authoring with peers, and talking about existing literature and academic service commitments can alleviate imposter syndrome. In this process, students can understand that they are most likely not alone in experiencing feelings of selfdoubt or confusion. Thus, talking to peers enables students to build relationships that allow them to ask sensitive questions and gradually develop a "successful scholarly identity" (Cassese and Holman 2018, 2). On the other hand, students who isolate themselves and communicate very little with their peers are likely to experience more feelings of self-doubt as they fail to recognize that their peers share these feelings. Through collective discussion, students can support one another and feel united through "common academic setbacks and negative thoughts affiliated with imposter syndrome" (Wilson and Cutri 2019, 71). Further, students should organize their time to schedule regular breaks to establish an effective work-life balance that does not lead to burnout (Hummel and Kurd 2021, 4). These peer relationships also provide invaluable opportunities to praise and elevate the work of those around you and to help them overcome their own feelings of imposter syndrome.

In a similar vein, mentors and advisers play a critical role. By regularly engaging (e.g., meeting once per month) with an assigned adviser, students can share their experiences with imposter syndrome, find support for their research, receive validation, and feel valued - all of which will positively impact a student's mental well-being (Evans et al. 2018, 283). Should a student lack an adviser who is supportive, they should contact the Graduate Director of their program to inquire about getting an additional or a new adviser. Students should have a supportive network of formal and informal advisers (Bothello and Roulet $2019,859)$ given the range of expertise available inside and outside of the political science discipline. For prospective and first-year students, they may be assigned an older student in the program who will act as a mentor, who they can likely speak to in confidence about their experiences with imposter syndrome. If this mentoring does not exist formally, older students will likely offer insights (informally) into their own experiences navigating imposter syndrome. This can help to manifest a sense of community and a sense of belonging that can subsequently diminish feelings of self-doubt. Supporting each other is key.

Furthermore, students can find value in their research. For some, it is engaging directly with communities affected by their research - whether as part of the research process, or separately in discussing their findings with them (Bothello and Roulet 2019, 859). Participating (attending and/or presenting) in political science conferences - like the American Political Science Association - can be great in further cultivating a sense of belonging by meeting like-minded scholars who share research interests. It is also important for graduate students to seek external validation outside of academia (Bothello and Roulet 2019, 860), as this can help to ground students in an understanding that there are important events occurring outside of the discipline of political science, and there is value in nonacademic or non-political science activity, too. 
Should opportunities arise for political science graduate students to advocate for departmental changes, there are a few encouraging ideas for enacting positive change. First, faculty ought to ensure that they regularly reach out to students, ideally once per month, to meet one-on-one with their mentees to provide ongoing support and openly discuss imposter syndrome and its associated effects. This is vital for students who belong to the groups that are more likely to experience imposter syndrome - first-gen, women, minorities, and international students. Second, advisers and mentors should allow their mentees to "wander intellectually and develop a unique identity as scholars," rather than pushing for students to narrowly confine themselves early in the process of becoming a political scientist (Bothello and Roulet 2019, 858).

Third, advisers should "teach their students to cope with work-related stress," "support helpseeking behaviors," and "help in linking-up to mental health professionals or counseling" services on- or off-campus (Muller 2020, 1280). For political science graduate students who have assistantships, they will likely possess some healthcare associated with their employment. In turn, they will have access to oncampus facilities like therapy services, and counseling and well-being centers. Also, advisers can draw attention to the positive aspects of imposter syndrome for those students already experiencing it, highlighting how self-awareness in the journey of becoming a political scientist is helpful for ongoing reflection (Tewfik 2021). Knowing the challenges that graduate students face with depression, anxiety and imposter syndrome, faculty should advocate for more resources and university support for mental health services. Finally, imposter syndrome is not universal and is not experienced the same for everyone, remember that you are not alone and there are others out there to help you in this journey.

\section{References}

Bothello, Joel., and Thomas J. Roulet. 2018. "The Imposter Syndrome, or the Mis-representation of Self in Academic Life.” Journal of Management Studies 56(4): 854-861. doi:

https://doi.org/10.1111/joms.12344

Cassese, Erin C., and Mirya R. Holman. 2018. "Writing Groups as Models for Peer Mentorship Among Female Faculty in Political Science.” PS: Political Science \& Politics 51(2): 401-405. doi: https://doi.org/10.1017/S1049096517002049

Evans, Teresa M., Lindsay Bira., Jazmin B. Gastelum., L. T. Weiss., and Nathan L. Vanderford. 2018. "Evidence for a Mental Health Crisis in Graduate Education." Nature Biotechnology 36(3): 282-284. doi: https://doi.org/10.1038/nbt.4089

Hummel, Calla., and Dana El Kurd. 2021. "Mental Health and Fieldwork." PS: Political Science \& Politics 54(1): 121-125. doi:10.1017/S1049096520001055

Mullangi, Samyukta., and Reshma Jagsi. 2019. "Imposter Syndrome: Treat the Cause, Not the Symptom.” Jama 322(5): 403-404. doi: 10.1001/jama.2019.9788

Müller, Astrid. 2020. "Mental Health Disorders: Prevalent but Widely Ignored in Academia?" Journal of Physiology 589(7): 1279-1281. doi: 10.1113/JP279386 
Benson, Thomas S., Bobbi G. Gentry., and Sarah Shugars

Rohrmann, Sonja., Bechtoldt, Myriam N., and Leonhardt, Mona. 2016. "Validation of the Imposter Phenomenon among Managers." Frontiers in Psychology 7: 821

https://doi.org/10.3389/fpsyg.2016.00821

Slank, Shanna. 2019. "Rethinking the Imposter Phenomenon.” Ethical Theory and Moral Practice: An International Forum 22(1): 205-218. doi: https://doi.org/10.1007/s10677-019-09984-8

Tewfik, B. 2021. "The Impostor Phenomenon Revisited: Examining the Relationship between Workplace Impostor Thoughts and Interpersonal Effectiveness at Work." Academy of Management Journal 0. doi: https://doi.org/10.5465/amj.2020.1627

Weir, Kirsten. 2013. “Feel like a Fraud?” American Psychological Association gradPSYCH 11(4). https://www.apa.org/gradpsych/2013/11/fraud.

Wilson, Sue., and Jennifer Cutri. 2019. "Negating Isolation and Imposter Syndrome Through Writing as Product and as Process: The Impact of Collegiate Writing Networks During a Doctoral Programme." In Wellbeing in Doctoral Education: Insights and Guidance from the Student Experience, eds. Lynette Pretorius, Luke Macaulay, and Basil Cahusac de Caux, 59-76. Cham, Switzerland: Springer. 